\title{
Effects of Coulomb interaction and tunneling on electron transport in coupled one-dimensional systems: from ballistic to diffusive regime.
}

\author{
O. E. Raichevt and P. Vasilopoulosd \\ Ðnst. of Semignonductor Physics, NAS Ukraine, Pr. Nauki 45, Kiev-28, 252650, Ukraine \\ - Concordia University, Montreal, Quebec H3G 1M8, Canada
}

\begin{abstract}
A linear theory of electron transport is developed for a system of two ideal quantum wires, of length $L$, coupled by tunneling and Coulomb interaction. The interaction of electrons with acoustical phonons is included and the results are valid in both the ballistic and diffusive regime. In the ballistic regime, both tunneling and Coulomb drag lead to a negative transresistance $R_{T R}$, while in the diffusive regime the tunneling opposes the drag and leads to a positive $R_{T R}$. If $L$ is smaller than the phase-breaking length, the tunneling leads to interference oscillations of the resistance that are damped exponentially with $L$.
\end{abstract}

PACS 73.40.Gk, 73.23.-b, 73.23.Ad

(August 10, 2018)

In submicrometer-long quantum wires and at low temperatures the electron transport is mainly ballistic ${ }^{1}$ and the wire conductance reaches its fundamental value of $G_{0}=e^{2} / \pi \hbar$. In contrast, for sufficiently long wires this transport is limited by scattering processes. The tunneling of electrons between parallel quantum wires and/or interlayer electron-electron (e-e) interaction are essential as demonstrated, e.g., by experimental and theoretical works on electron transport ${ }^{2-13}$ along the layers in coupled double-wire systems. These works are mostly devoted to studying interlayer tunneling in the purely ballistic regime ${ }^{2-10}$ or momentum transfer between the wires (Coulomb drag) ${ }^{11-13}$. The drag effect has been studied both in the diffusive ${ }^{11}$ and ballistic ${ }^{12}$ transport regimes as well as when the electrons are described by a Luttinger liquid ${ }^{13}$.

Despite this progress, the description of electron transport in coupled quantum wires is substantially insufficient. Even within the concept that the electrons are described by a normal Fermi liquid, two important questions arise. The first one is how to describe the transport properties when both tunneling and the interactions of electrons with each other, and with impurities or phonons, are essential. The second question is how to bridge the gap between the ballistic and diffusive regimes in such a description. In this Letter we present a linearresponse theory of electron transport in coupled quantum wires that gives a reasonable answer to both questions and the new results mentioned in the abstract.

From kinetic theory to local description. We consider two parallel, tunnel-coupled 1D layers of degenerate electrons adiabatically contacted to equilibrium reservoirs (leads) as shown and labelled in Fig. 1. We start from the quantum kinetic equation $\partial \hat{\rho} / \partial t+(i / \hbar)\left[\hat{H}_{0}+\right.$ $\left.\hat{H}_{C}+\hat{H}_{e-p h}, \hat{\rho}\right]=0$ for the density matrix $\hat{\rho}$ and assume that electrons interact with the Coulomb field $\left(\hat{H}_{C}\right)$ and with acoustical phonons $\left(\hat{H}_{e-p h}\right)$. Elastic scattering is neglected, i.e., we assume ideal wires. We employ a tight-binding description with basis that of the isolated left $(l)$ and right $(r)$ layer states in which the potential energy matrix is $\hat{h}=(\Delta / 2) \hat{\sigma}_{z}+T \hat{\sigma}_{x}$ when only the lowest subband is occupied in either layer. Here $\hat{\sigma}_{i}$ are the Pauli matrices, $\Delta$ is the level splitting energy, and $T$ the tunneling matrix element.

The kinetic equation can be written ${ }^{14}$ as one for the Keldysh's Green's function $\hat{G}^{-+}$. Below we consider the case when the characteristic spatial scale of the electronic distribution is large in comparison with the Fermi wavelength $\pi \hbar / p_{F}$ and use the Keldysh's matrix Green's function in the Wigner representation $\hat{G}_{\varepsilon, t}^{-+}(p, x)$, where $p$ and $\varepsilon$ are the momentum and energy. In the steady-state regime the time-averaged Green's functions are linearized in the manner $\hat{G}_{\varepsilon}^{\alpha \beta}(p, x)=\hat{G}_{\varepsilon}^{(0) \alpha \beta}(p)+\delta \hat{G}_{\varepsilon}^{\alpha \beta}(p, x)$, where $\alpha$ and $\beta$ stand for + or - . The unperturbed part $\hat{G}_{\varepsilon}^{(0) \alpha \beta}(p)$ is expressed through the retarded and advanced matrix Green's functions and the equilibrium Fermi-Dirac function $f(\varepsilon)=1 /\left[1+e^{(\varepsilon-\mu) / k_{B} T_{e}}\right]$ in the usual way ${ }^{14}$. The linearized kinetic equation reads

$$
\begin{gathered}
\frac{\hbar}{2}\left\{\hat{v}_{p}, \frac{\partial}{\partial x} \delta \hat{G}_{\varepsilon}^{-+}(p, x)\right\}+i\left[\hat{h}, \delta \hat{G}_{\varepsilon}^{-+}(p, x)\right]+i\left[\hat{\varphi}, \hat{G}_{\varepsilon}^{(0)-+}(p)\right] \\
-\frac{\hbar}{2}\left\{\frac{\partial}{\partial x} \hat{\varphi}, \frac{\partial}{\partial p} \hat{G}_{\varepsilon}^{(0)-+}(p)\right\}=i \delta \hat{\mathcal{I}}(\varepsilon, p, x) .
\end{gathered}
$$

Here $\{\ldots\}$ denotes anticommutators, $\hat{v}_{p}=\hat{P}_{l} v_{l p}+\hat{P}_{r} v_{r p}$ is the diagonal matrix of the group velocities, and $\hat{P}_{l}$ and $\hat{P}_{r}$ are the projection matrices. We consider only the case of equal group velocities in the layers, when $v_{l p}=v_{r p}=v_{p}=p / m$. Further, $\hat{\varphi}$ is the matrix of the self-consistent electrostatic potential resulting from the perturbation of the electron density. The collision integral is given as ${ }^{14} \hat{\mathcal{I}}=-\left[\hat{\Sigma}^{-+} \hat{G}^{++}+\hat{\Sigma}^{--} \hat{G}^{-+}+\right.$ $\left.\hat{G}^{-+} \hat{\Sigma}^{++}+\hat{G}^{--} \hat{\Sigma}^{-+}\right]$, where $\hat{\Sigma}^{\alpha \beta}$ is the self-energy due to Coulomb and electron-phonon interactions, and where the arguments of all functions are $\varepsilon, p$, and $x$. This corresponds to a quasiclassical description of the scattering. Despite the approximations made, Eq. (1), due to its matrix structure, is not reduced to a classical Boltzmann 
equation.

We assume that the Fermi energy is large in comparison with both $T$ and $\Delta$. We sum up Eq. (1) over the electron momentum $p$ in the regions of forward $(+)$ and backward (-) group velocities and introduce the nonequilibrium part of the energy distribution function $\hat{g}_{\varepsilon}(x)$

$$
\hat{g}_{\varepsilon}^{ \pm}(x)=(1 / 2 \pi i) \int_{ \pm} d p\left|v_{p}\right| \delta \hat{G}_{\varepsilon}^{-+}(p, x) .
$$

Since $\delta \hat{G}^{-+}$is essentially nonzero only in narrow intervals of energy and momentum near the chemical potential $\mu$ and Fermi momentum, we can replace $\left|v_{p}\right|$ by the Fermi velocity $v_{F}$ common to both layers. The result is

$$
\pm v_{F} \partial \hat{g}_{\varepsilon}^{ \pm}(x) / \partial x+(i / \hbar)\left[\hat{h}, \hat{g}_{\varepsilon}^{ \pm}(x)\right]=\delta \hat{I}_{ \pm}(\varepsilon, x)
$$

where $\delta \hat{I}_{ \pm}(\varepsilon, x)=(1 / 2 \pi \hbar) \int_{+}\left|v_{p}\right| d p \delta \hat{\mathcal{I}}(\varepsilon, p, x)$ depends on both $\hat{g}^{+}$and $\hat{g}^{-}$since it accounts for both forwardand backscattering processes. Notice that the absence of $\hat{\varphi}$ in Eq. (3) is not a simplification or an approximation: it results exactly from the integration of Eq. (1) over momentum.

The matrix kinetic equation (3) is equivalent to eight scalar equations for the four components of $\hat{g}^{+}$and those (four) of $\hat{g}^{-}$. The boundary conditions for them are determined, in the Landauer-Büttiker-Imry approach, by the distribution functions of the leads described by four chemical potentials $\mu_{1 l}, \mu_{1 r}, \mu_{2 l}$, and $\mu_{2 r}$, so that $\hat{g}_{\varepsilon}^{+}(0)=-(\partial f(\varepsilon) / \partial \varepsilon)\left[\hat{P}_{l} \delta \mu_{1 l}+\hat{P}_{r} \delta \mu_{1 r}\right]$, and $\hat{g}_{\varepsilon}^{-}(L)=-(\partial f(\varepsilon) / \partial \varepsilon)\left[\hat{P}_{l} \delta \mu_{2 l}+\hat{P}_{r} \delta \mu_{2 r}\right]$. The forward- and backward-propagating states are "connected" to leads "1" and "2", respectively. The nondiagonal components vanish at the contacts since the tunneling is absent beyond the region $x=[0, L]$.

We now introduce the local chemical potential matrix $\delta \hat{\mu}^{ \pm}(x)=\hat{P}_{l} \delta \mu_{l}^{ \pm}(x)+\hat{P}_{r} \delta \mu_{r}^{ \pm}(x)+\hat{\sigma}_{x} \delta \mu_{x}^{ \pm}(x)+\hat{\sigma}_{y} \delta \mu_{y}^{ \pm}(x)$ according to $\delta \hat{\mu}^{ \pm}(x)=\int d \varepsilon \hat{g}_{\varepsilon}^{ \pm}(x)$. Assuming that backscattering occurs much less frequently than forwardscattering, which is the case when the e-e scattering dominates over the electron-phonon one, and that tunneling also occurs much less frequently than forward-scattering, we can write $\left[\hat{g}_{\varepsilon}^{ \pm}(x)\right]_{l l, r r}=-(\partial f(\varepsilon) / \partial \varepsilon) \delta \mu_{l, r}^{ \pm}(x)$. Below we omit the symbol " $\delta$ " in $\delta \hat{\mu}^{ \pm}(x)$ and in the potentials of the leads since all potentials are measured from the same equilibrium value $\mu$. Then integrating Eq. (3) over the energy we obtain eight coupled, first-order differential equations:

$$
\begin{array}{r} 
\pm d \mu_{l}^{ \pm} / d x+\left(\mu_{l}^{ \pm}-\mu_{l}^{\mp}\right)\left(1 / l_{P}+1 / l_{D}\right) \\
-\left(\mu_{r}^{ \pm}-\mu_{r}^{\mp}\right) / l_{D}-2 t_{F} \mu_{y}^{ \pm}=0, \\
\pm d \mu_{r}^{ \pm} / d x+\left(\mu_{r}^{ \pm}-\mu_{r}^{\mp}\right)\left(1 / l_{P}+1 / l_{D}\right) \\
-\left(\mu_{l}^{ \pm}-\mu_{l}^{\mp}\right) / l_{D}+2 t_{F} \mu_{y}^{ \pm}=0, \\
\pm d \mu_{x}^{ \pm} / d x+\delta_{F} \mu_{y}^{ \pm}+\mu_{x}^{ \pm} / l_{C}=0, \\
\pm d \mu_{y}^{ \pm} / d x-\delta_{F} \mu_{x}^{ \pm}+\mu_{y}^{ \pm} / l_{C}+t_{F}\left(\mu_{l}^{ \pm}-\mu_{r}^{ \pm}\right)=0 .
\end{array}
$$

Here $t_{F}=T / \hbar v_{F}, \delta_{F}=\Delta / \hbar v_{F}$. The evaluation of $\delta \hat{I}$ was carried out assuming a weak tunnel coupling, when $T$ is small in comparison to the imaginary part of the selfenergies. The characteristic lengths $l_{P}, l_{D}$ and $l_{C}$, resulting from the collision integral $\delta \hat{I}_{ \pm}(\varepsilon, x)$, are expressed, respectively, through the phonon-assisted $1 \mathrm{D}$ transport time $^{15} \tau_{P}$, the $1 \mathrm{D}$ Coulomb drag time ${ }^{11} \tau_{D}$, and the phase-breaking time $\tau_{C}$ (which describes the suppression of tunnel coherence) as $l_{P}=2 v_{F} \tau_{P}, l_{D}=2 v_{F} \tau_{D}$, and $l_{C}=v_{F} \tau_{C}$. All these times are microscopically justified. For $\tau_{C}$, if we account only for the lowest-order Coulomb contributions, we obtain the new result

$$
1 / \tau_{C} \simeq\left[e^{4} \ln ^{2}(w / a) \Delta / 2 \pi \hbar^{3} \epsilon^{2} v_{F}^{2}\right] \operatorname{cotanh}\left(\Delta / 4 k_{B} T_{e}\right)
$$

where $\epsilon$ is the dielectric constant, $w$ is the distance between the wires and $a$ the wire width. Estimating $\tau_{C}$ from Eq. (8), we find $l_{C} \ll l_{P}$ and $l_{C} \ll l_{D}$, because of the weakness of the electron-phonon coupling and of the e-e backscattering, respectively.

Equations (4)-(7) with the boundary conditions $\mu_{l}^{+}(0)=\mu_{1 l}, \mu_{l}^{-}(L)=\mu_{2 l}, \mu_{r}^{+}(0)=\mu_{1 r}, \mu_{r}^{-}(L)=\mu_{2 r}$ and $\mu_{x, y}^{+}(0)=\mu_{x, y}^{-}(L)=0$ give a complete description of electron transport in double quantum wire systems in a wide range of regimes starting from the purely ballistic regime, for $L \ll l_{C}$, to the diffusive regime, for $L \gg l_{P}, l_{D}$. The local currents flowing in the layers $j=l, r$ are expressed by the Landauer-like formula

$$
J_{j}(x)=G_{0}\left[\mu_{j}^{+}(x)-\mu_{j}^{-}(x)\right] / e .
$$

Below, to characterize the effects of drag and tunneling, we calculate the transresistance $R_{T R}=\left[\mu_{1 l}-\mu_{2 l}\right] / e J_{r}$, where $J_{r}$ is the current injected in the wire $r$ when no current is allowed to flow into wire $l$. This is the typical setup for the drag experiments. In this case $J_{r}(0)=$ $J_{r}(L)=J_{r}$ and $J_{l}(0)=J_{l}(L)=0$. We also calculate the direct resistance $R=\left[\mu_{1 r}-\mu_{2 r}\right] / e J_{r}$.

Results. The solution of Eqs. (4)-(7) is obtained for all potentials in the form of $A+B x+\sum_{ \pm} C_{i}^{ \pm} e^{ \pm \lambda_{i} x}$, where $\lambda_{i}^{2}$ are the roots of a cubic equation. Explicitly, for $l_{C} \ll l_{P}, l_{D}$ and weak tunnel coupling $t_{F} \ll l_{C}^{-1}$, the three roots are $\lambda_{1}=\lambda \simeq 2\left(1 / l_{T}^{2}+1 / l_{T} l_{1}\right)^{1 / 2}$ and $\lambda_{2,3}=\lambda_{ \pm} \simeq 1 / l_{C} \pm i \delta_{F}$, where $1 / l_{1}=1 / l_{P}+2 / l_{D}$ and $l_{T}=v_{F} \tau_{T}$. The tunneling time $\tau_{T}$ is given by $1 / \tau_{T}=\left(1 / \tau_{C}\right) 2 T^{2} /\left(\Delta^{2}+\hbar^{2} / \tau_{C}^{2}\right)$. The terms with $\lambda$ describe long-scale variations of the chemical potentials, while those with $\lambda_{ \pm}$correspond to short-scale variations. Two cases can be considered.

Long wires, $L \gg l_{C}$. The solutions containing $\lambda_{ \pm}$are not essential in the calculation of the currents. Considering only the solutions with $\lambda$, we find

$$
\begin{array}{r}
R=\left(\pi \hbar / 2 e^{2}\right)\left[2+L / l_{P}+\sqrt{1+l_{T} / l_{1}} \tanh (\lambda L / 2)\right] \\
R_{T R}=\left(\pi \hbar / 2 e^{2}\right)\left[L / l_{P}-\sqrt{1+l_{T} / l_{1}} \tanh (\lambda L / 2)\right] .
\end{array}
$$

In the ballistic regime $\left(L \ll l_{P}, l_{T}\right)$ we obtain the usual resistance $R \simeq \pi \hbar / e^{2}=G_{0}^{-1}$, while the transresistance is 
given by $R_{T R}=-\left(\pi \hbar / e^{2}\right) L\left[1 / l_{D}+1 / 2 l_{T}\right]$. As seen, $R_{T R}$ is small, always negative, and proportional to $L$. If we neglect tunneling, the resulting $R_{T R}$ describes the Coulomb drag in the ballistic regime ${ }^{12}$. In the diffusive regime, i.e., when $L$ increases and the relation $L \gg l_{P}$ holds, we obtain $R \simeq\left(\pi \hbar / e^{2}\right) L\left[1 / l_{P}+1 / l_{D}\right]$ for $\lambda L / 2 \ll 1$. This resistance, if one omits the drag contribution, is expressed in terms of the Drude conductivity $\sigma=L / R=$ $e^{2} l_{P} / \pi \hbar=e^{2} n \tau_{P} / m$, where $n$ is the $1 \mathrm{D}$ electron density in layer $r$. Then $R_{T R}=-\left(\pi \hbar / e^{2}\right)\left(L / l_{D}\right)\left[1-\left(L / L_{0}\right)^{2}\right]$ where $L_{0}=\left(6 l_{P}^{2} l_{T} / l_{D}\right)^{1 / 2}$. Introducing the drag transresistivity $\pi \hbar / e^{2} l_{D}$ and the 1D-1D tunneling conductance $e^{2} \rho_{1 D} / \tau_{T}=2 e^{2} / \pi \hbar l_{T}$, where $\rho_{1 D}$ is the $1 \mathrm{D}$ density of states at the Fermi level, we formally obtain the result of Ref. 16 where a competition of drag and tunneling was investigated, for double quantum well systems, only in the diffusive regime. As the factor $\left[1-\left(L / L_{0}\right)^{2}\right]$ shows, in this regime the tunneling opposes the drag and the transresistance increases, changing its sign from negative to positive. If $\lambda L / 2 \sim 1, R_{T R}$ is large and always comparable to $R$, because a considerable fraction of the current penetrates in the $l$ layer due to tunneling. In Fig. 2 we show the length dependence of $R_{T R}$ given by Eq. (11) for different relative contributions of the Coulomb drag and tunneling. $R_{T R}$ is negative for small $L$ but it always changes sign and becomes positive as $L$ increases. This occurs at smaller $L$ when the tunneling is stronger (larger $l_{P} / l_{T}$ ) or the drag is weaker ( $\operatorname{smaller} l_{P} / l_{D}$ ).

A peculiar transport regime, without backscattering, can be realized in tunnel-coupled magnetic edge states $^{17,18}$. Assuming $1 / l_{P}=1 / l_{D}=0$ in Eqs. (10) and (11), we obtain the result of Ref. 18 in the form $R=\left(\pi \hbar / e^{2}\right)\left[1+(1 / 2) \tanh \left(L / l_{T}\right)\right]$ and $R_{T R}=$ $-\left(\pi \hbar / 2 e^{2}\right) \tanh \left(L / l_{T}\right)$.

Short wires $L \sim l_{C} \ll l_{P}, l_{D}$. Electrons pass through the wires almost without backscattering, $R \simeq \pi \hbar / e^{2}$, and $R_{T R}$ is small. However, an electron tunneling between the layers does not lose its phase memory completely; as a result tunnel coherence effects can take place and give additional contributions to $R$ and $R_{T R}$ :

$$
\begin{array}{r}
R=\frac{\pi \hbar}{e^{2}}\left[1+\frac{L}{l_{P}}+\frac{L}{l_{D}}+\frac{L}{2 l_{T}}-\frac{l_{C}}{2 l_{T}} \Phi(L)\right], \\
R_{T R}=\frac{\pi \hbar}{e^{2}}\left[-\frac{L}{l_{D}}-\frac{L}{2 l_{T}}+\frac{l_{C}}{2 l_{T}} \Phi(L)\right],
\end{array}
$$

where

$$
\begin{aligned}
\Phi(L)= & \left(l_{C}^{-2}+\delta_{F}^{2}\right)^{-1}\left[2\left(\delta_{F} / l_{C}\right) e^{-L / l_{C}} \sin \left(\delta_{F} L\right)\right. \\
& \left.+\left(l_{C}^{-2}-\delta_{F}^{2}\right)\left(1-e^{-L / l_{C}} \cos \left(\delta_{F} L\right)\right)\right] .
\end{aligned}
$$

The term proportional to $\Phi(L)$ describes oscillations of the resistance damped due to the factor $\exp \left(-L / l_{C}\right)$. Its relative contribution to $R_{T R}$ is not small at $L \sim l_{C}$. The periodic behavior can be described as a result of tunneling-assisted interference of electron waves of the left and right layers on the coupling length $L$. Due to a finite $\Delta$, these waves have slightly different phase velocities. Figure 3 shows the dependence of $\Phi(L)$ as a function of $\delta_{F} L$; we used Eq. (8) at zero temperature so that $1 / l_{C}=\kappa\left|\delta_{F}\right|$, where $\kappa$ is a dimensionless parameter.

The level splitting $\Delta$ can be changed not only by a transverse voltage, but also by a magnetic field ${ }^{9} H$ perpendicular to the plane of the quantum wires. For sufficiently weak $H$ the results presented so far still hold with the phase $\delta_{F} L$ in Eq. (14) changed to $\delta_{F} L+2 \pi \phi / \phi_{0}$, where $\phi_{0}=h / e$ is the magnetic flux quantum and $\phi=H w L$ the flux enclosed by the area between the wires. Though the double-wire system does not form a closed current loop, this should lead to Aharonov-Bohmtype oscillations in $R$ and $R_{T R}$.

In very short wires, with $L \ll l_{C}, \delta_{F}^{-1}$, we have $R_{T R}=$ $\left(\pi \hbar / e^{2}\right)\left[-L / l_{D}-L^{2} t_{F}^{2} / 2\right]$ : in contrast with the drag contribution, that of tunneling shows a $L^{2}$ dependence.

Finally, we apply Eq. (3) to the purely ballistic regime in which we can neglect the collision integral and need not make the assumption of weak tunnel coupling. Then we obtain Eqs. (4)-(7) without the terms containing $l_{P}, l_{D}$ and $l_{C}$; they describe oscillations of the electronic wave packets between the layers due to coherent tunneling. Solving them, we obtain

$$
\begin{gathered}
R=\left(\pi \hbar / e^{2}\right)\left[1-r \sin ^{2} \psi\right]\left[1-2 r \sin ^{2} \psi\right]^{-1}, \\
R_{T R}=-\left(\pi \hbar / e^{2}\right) r \sin ^{2} \psi\left[1-2 r \sin ^{2} \psi\right]^{-1},
\end{gathered}
$$

where $r=2 T^{2} / \Delta_{T}^{2}, \Delta_{T}=\left(\Delta^{2}+4 T^{2}\right)^{1 / 2}$ and $\psi=$ $\Delta_{T} L / 2 \hbar v_{F}$. The periodicity of the oscillations becomes the same as in Eqs. (12) and (13) if one replaces $\Delta$ by $\Delta_{T}$. However, since the tunnel coupling is not weak, the oscillations described by Eqs. (15) and (16) have large amplitudes. In particular, when $\Delta$ is small, both $R$ and $R_{T R}$ show giant oscillations with amplitude large in comparison with $1 / G_{0}$. The experimentally observed ${ }^{6}$ resistance oscillations, resulting from tunnel coupling over a finitelength region, in ballistic quantum wires were of small $(\sim 0.5 \mathrm{~K} \Omega)$ amplitude. This is not surprising because there are many factors, e.g., long-scale inhomogeneities, inelastic and elastic scattering, which compete against the tunnel coherence. Previous theoretical studies of the purely ballistic transport regime ${ }^{2-5,7-9}$ were based on a quantum-mechanical calculation of the electronic wave transmission, while we recover the essential results from a quantum-kinetic analysis.

In conclusion, we developed a linear-response, steadystate theory of electron transport in parallel onedimensional layers coupled by tunneling and Coulomb interaction and contacted to quasi-equilibrium reservoirs. The quantum-kinetic description of the problem leads to linear differential equations describing the distribution of local chemical potentials of the forward- and backwardmoving electrons. The results for the direct resistance $R$ and the transresistance $R_{T R}$ are valid in the whole range from the pure ballistic to the diffusive regime. $R_{T R}$ always depends nonmonotonically on the wire length $L$ : in the ballistic regime tunneling and drag lead to a negative 
transresistance $R_{T R}$, while in the diffusive regime the tunneling opposes the drag and leads to a positive $R_{T R}$. When $L$ is smaller than the phase-breaking length, the tunneling leads to interference oscillations of the resistance damped exponentially with $L$.

This work was supported by the Canadian NSERC Grant No. OGP0121756.

$\dagger \quad$ E-mail: zinovi@lab2.kiev.ua

$\diamond \quad$ E-mail: takis@boltzmann.concordia.ca

[1] S. Datta, Electronic Transport in Mesoscopic Systems, Cambridge Univ. Pr. (1997).

[2] J. A. del Alamo and C. C. Eugster, Appl. Phys. Lett. 56, 78 (1990).

[3] N. Tsukada et al., Appl. Phys. Lett. 56, 2527 (1990).

[4] R. Q. Yang and J. M. Xu, Phys. Rev. B 43, 1699 (1991).

[5] P. Zhao, Phys. Rev. B 45, 4301 (1992).

[6] M. Okuda et al., Phys. Rev. B 47, 4103 (1993).

[7] J.-Q. Wang and B.-y. Gu, Phys. Rev. B 47, 13442 (1993).

[8] N. Tsukada et al., Phys. Rev. B 53, R7603 (1996).

[9] J.-R. Shi, and B.-Y. Gu, Phys. Rev. B 55, 9941 (1997).

[10] I. M. Castleton et al., Physica B, 249-245, 157 (1998).

[11] Yu. M. Sirenko and P. Vasilopoulos, Phys. Rev. B 46, 1611 (1992).

[12] V. I. Gurevich et al., J. Phys.: Cond. Matter 10, 2551 (1998).

[13] K. Flensberg, Phys. Rev. Lett. 81, 184 (1998).

[14] see E. M. Lifshitz and L. P. Pitaevski, Physical Kinetics, Pergamon Press, Oxford, 1981.

[15] J. Lee and M. O. Vassel, J. Phys. C 172525 (1984).

[16] O. E. Raichev, J. Appl. Phys. 81, 1302 (1997).

[17] Y. Ohno et al., Phys. Rev. B 54, R2319 (1996).

[18] D. Yoshioka and A. H. MacDonald, Phys. Rev. B 53, R16168 (1996). 


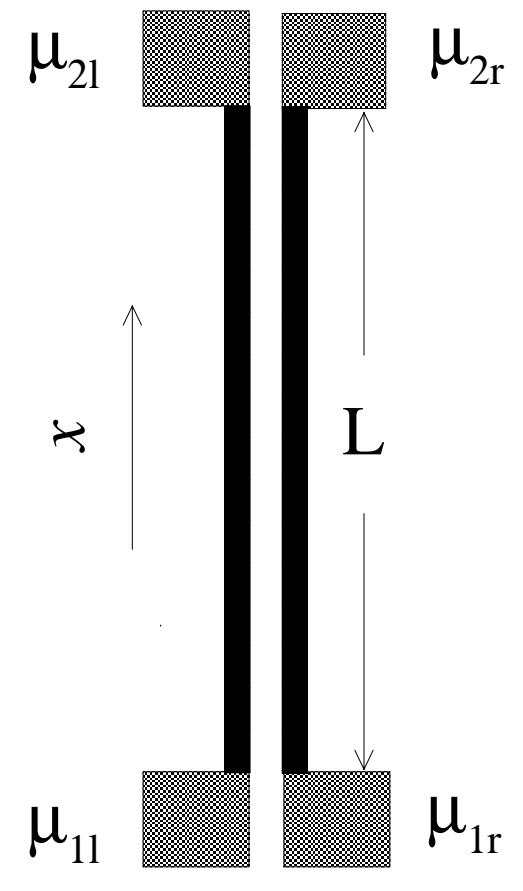

Fig.1 O.E.Raichev and P.Vasilopoulos, Effects of.. 


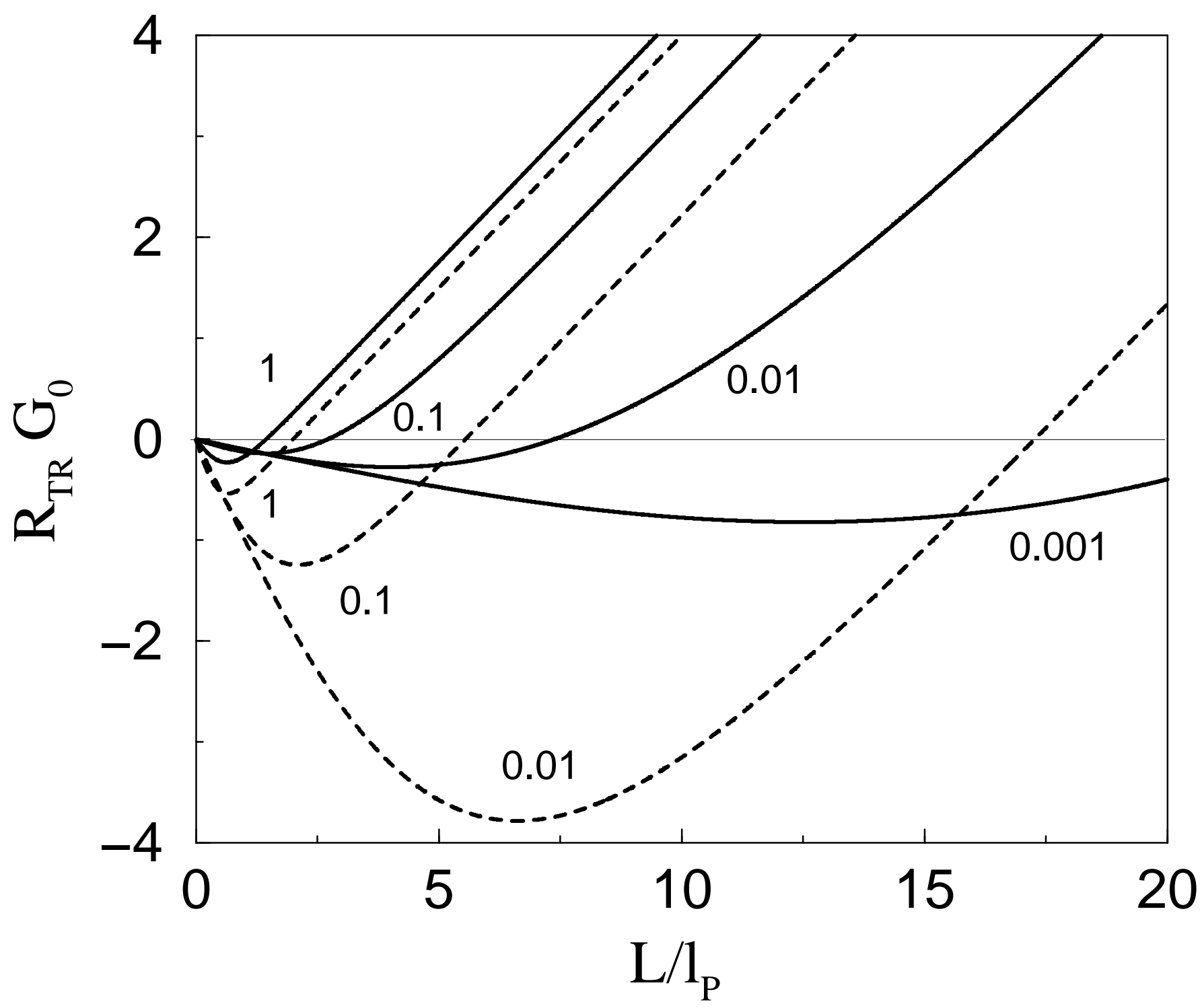

Fig.2 O.E.Raichev and P.Vasilopoulos, Effects of... 


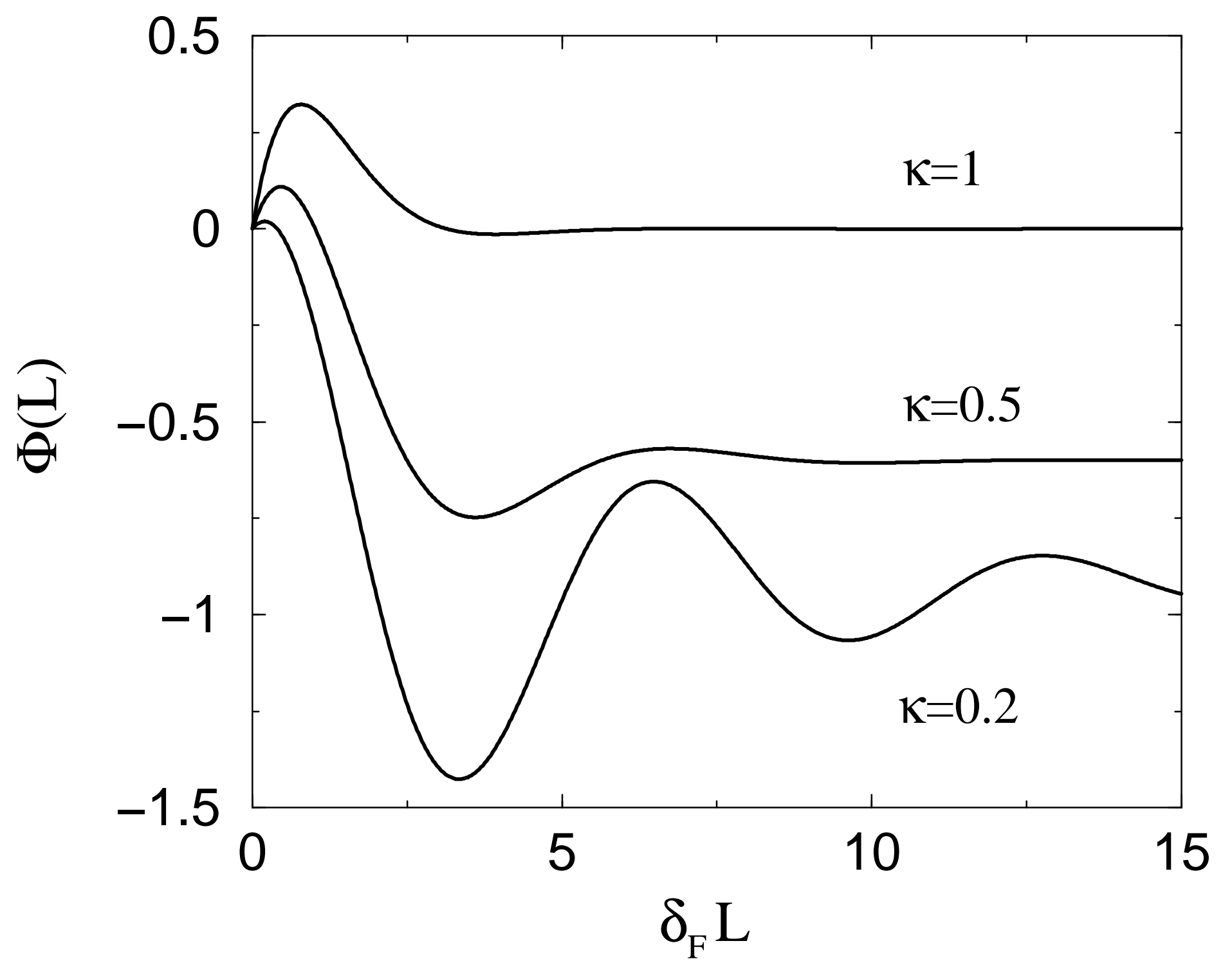

Fig. 3 O.E Raichev and P.Vasilopoulos, Effects of... 